\title{
Psoriatic arthritis: clinical subgroups and histocompatibility antigens
}

\author{
N J McHUGH, ${ }^{1}$ M R LAURENT ${ }^{1}$ B L J TREADWELL, ${ }^{1}$ J M TWEED, ${ }^{1}$ \\ AND J DAGGER
}

From the ${ }^{1}$ Wellington Regional Rheumatic Diseases Unit, Lower Hutt, and the ${ }^{2}$ Immunohaematolog Laboratory, Wellington Hospital, New Zealand

SUMMARY Histocompatibility antigens were determined in 60 patients with psoriatic arthritis The patients were divided into clinical subgroups according to axial or peripheral joini $\dot{\infty}_{\infty}$ involvement, disease severity based on number of peripheral joints involved, and the presence or absence of bone erosions. The total group showed a significant increase in frequency of HLA-A1? B17, B27, and DR7 when compared with a control population. The subgroup with spondylitis had a significant increase in frequency of HLA-B27 when compared with patients with periphera林 arthritis $(\mathrm{p}<0 \cdot 001)$. The subgroup with peripheral arthritis alone had a higher frequency of HLA $)$ DR7 than the control group $(p<0.001)$. There were also significant associations between HLA $\vec{\varpi}$ DR7 and chronic severe disease $(p<0 \cdot 001)$ and between HLA-DR4 and the presence of erosions $(\mathrm{p}<0 \cdot 05)$.

Key words: psoriasis, HLA antigens, spondylitis.

The genetic predisposition to psoriasis and psoriatic arthritis has been the subject of many studies. There have been a number of reported associations with major histocompatibility complex class I antigens, the most significant being the association of psoriatic spondylitis with HLA-B27. ${ }^{\prime}$ In patients with psoriasis there is an increased frequency of HLA-A1, $\mathrm{B} 13, \mathrm{~B} 17$, and $\mathrm{Cw} 6,{ }^{2-6}$ and in those with arthritis an increase in HLA-A26, B27, B38, B39, and Cw6, ${ }^{7-14}$ HLA-B38 being associated with a peripheral arthritis. ${ }^{8}$

The association with class II antigens is more controversial. HLA-DR4 has been associated with seropositive rheumatoid arthritis, ${ }^{15}$ ankylosing spondylitis with peripheral joint involvement, ${ }^{16}$ and occasionally psoriatic arthritis. ${ }^{8-10}$ In psoriatic arthritis HLA-DR4 may be associated with more severe arthritis ${ }^{10}$ and HLA-DR3 with the presence of erosions. ${ }^{11}$ HLA-DR7 is increased in psoriatic arthritis $^{8911} 13$ more commonly than in psoriasis alone. ${ }^{11}$ The association between HLA-DR antigens and psoriatic arthritis and its subgroups remains

Accepted for publication 17 September 1986.

Correspondence to Dr N J McHugh, Royal National Hospital for Rheumatic Diseases, Upper Borough Walls, Bath BA1 1RL. controversial. The aim of this study was to investiog gate these associations further.

\section{Patients and methods}

Sixty unrelated Caucasian patients (29 male, 3항 female) attending a rheumatology clinic in thẹ . Wellington area were studied. They all had psoriatis arthritis according to the criteria of Moll anळ Wright $^{17}$ and were seronegative for IgM and IgC rheumatoid factor. All patients had radiographs of clinically affected joints. Thus all patients has radiographs of hands and feet, apart from four who had no clinical evidence of peripheral joint involvee ment. Thirty seven patients had radiographs of scaroiliac joints, including 13 from the group who had arthritis limited to peripheral joints on clinicat examination. HLA-A and B antigens were deter mined by a standard lymphocytotoxicity method and HLA-DR antigens using prolonged lymphocytos toxicity on B lymphocytes separated by nylon woo $\bar{b}$ Three hundred and seventy five Caucasian bloo 6 donors and laboratory staff were used as controls foop class I antigens and 163 Caucasian blood donors an laboratory staff were used as controls for class antigens. 
The patients were divided into the following subgroups: (1) peripheral arthritis only (PAO); (2) peripheral arthritis with or without axial disease but not fulfilling Rome criteria for spondylitis ${ }^{18}$ (i.e., axial involvement being asymptomatic sacroiliitis or cervical spine involvement (PA $\pm A x-S P)$; (3) axial disease (spondylitis or sacroiliitis, or both) with or without peripheral arthritis (and not necessarily fulfilling Rome criteria) ( $\mathrm{Ax} \pm \mathrm{PA})$; (4) spondylitis (Rome criteria) with or without peripheral arthritis (SP \pm PA); (5) spondylitis only (Rome criteria without peripheral joint involvement (SPO); (6) cervical spine involvement (clinical or radiological) (CS).

The sum of group 1 and its control group 3 represents the total group as does the sum of group 2 and its control group 4.

They were also divided into three groups according to disease severity as described by Gerber et al. ${ }^{10}$ This was based on the number of joints involved either clinically or radiologically, excluding the vertebral column from the joint count, and assessed as mild (fewer than 10 joints), moderate (10-20 joints), or severe (more than 20 joints). Duration of disease was also recorded.

Patients with bone erosions were compared with those without erosions.

Statistical analysis was by the $\chi^{2}$ test with Yates's correction. $p$ Values were corrected for the number of variables tested when assessing any previously unreported findings.

\section{Results}

In the total patient group there was a significant increase in HLA-A1 $(\mathrm{p}<0.02), \mathrm{B} 17(\mathrm{p}<0.02), \mathrm{B} 27$ $(p<0 \cdot 001)$, and DR7 $(p<0 \cdot 01)$ (Table 1). HLA-B38 and HLA-B39 were not increased in either the total group or in those with peripheral arthritis. There was a highly significant increase of HLA-DR7 in the group with peripheral arthritis $(p<0 \cdot 001)$.

The association of HLA-B27 with spondylitis or axial disease was highly significant compared with both the control population and with those with peripheral arthritis alone. The four patients who had spondylitis only were all male and all HLA-B27 positive. HLA-B27 was not significantly increased in peripheral disease (Table 1).

There were six patients with peripheral arthritis and axial involvement who did not fulfil the criteria of spondylitis, three with cervical spine involvement and three with unilateral sacroiliitis. Only one of this group with unilateral sacroiliitis was HLA-B27 positive.

There were eight patients with significant cervical spine involvement clinically or radiologically. There may have been others with subclinical involvement who were not $x$ rayed. Six of the eight were HLADR1 positive. Three of the eight, all female, did not meet the criteria for spondylitis, yet had severe cervical spine involvement with ankylosis and peripheral joint disease, and their sacroiliac joints were radiologically normal. These three were HLAB27 negative. The other five had clinical and radiological spondylitis, four were HLA-B27 positive, and one of these had normal sacroiliac joints.

The group with severe peripheral arthritis had an increased frequency of HLA-A1, B17, and DR7 when compared with the control population, and

Table 1 HLA antigens and psoriatic arthritis (results expressed as a percentage)

\begin{tabular}{|c|c|c|c|c|c|c|c|c|}
\hline & Control & Total & $P A O_{\dagger}^{\dagger}$ & $P A \pm A x-S P$ & $A x \pm P A$ & $S P \pm P A$ & $S P O$ & $C S$ \\
\hline No & 375 & 60 & 33 & 39 & 27 & 21 & 4 & 8 \\
\hline Male/female & & $29 / 31$ & $15 / 18$ & $17 / 22$ & $14 / 13$ & $12 / 9$ & $4 / 0$ & $2 / 6$ \\
\hline A1 & $33 \cdot 6$ & $50^{*}$ & & & & & & \\
\hline A26 & $3 \cdot 2$ & $6 \cdot 7$ & & & & & & \\
\hline B7 & $27 \cdot 2$ & $13 \cdot 3$ & $21 \cdot 2$ & $20 \cdot 5$ & $3 \cdot 7^{* *}$ & $0 \div \div$ & & \\
\hline B17 & $11 \cdot 2$ & $23 \cdot 3^{*}$ & & & & & & \\
\hline B27 & $7 \cdot 7$ & $31 \cdot 7^{* * *}$ & $18 \cdot 2$ & $15 \cdot 4$ & $48 \cdot 1^{* * *}+$ & $61 \cdot 9^{* * *+\dagger}$ & 100 & 50 \\
\hline B38 & $1 \cdot 0$ & 5 & 0 & $5 \cdot 1$ & $11 \cdot 1$ & $4 \cdot 7$ & & \\
\hline B39 & $2 \cdot 1$ & 5 & & & & & & \\
\hline No & 163 & 57 & 31 & 37 & 26 & 20 & 4 & 8 \\
\hline DR 1 & $31 \cdot 2$ & $24 \cdot 6$ & & & & & & 75 \\
\hline DR2 & $25 \cdot 1$ & 14 & & & & & & \\
\hline DR3 & $27 \cdot 6$ & $12 \cdot 3$ & & & & & & \\
\hline DR4 & $28 \cdot 8$ & $36 \cdot 8$ & $41 \cdot 9$ & $35 \cdot 1$ & $30 \cdot 7$ & 40 & & \\
\hline DR7 & $22 \cdot 6$ & $43 \cdot 9^{* *}$ & $51 \cdot 6^{* * *}$ & $48 \cdot 6^{* *}$ & $34 \cdot 6$ & 35 & & \\
\hline
\end{tabular}

${ }^{*} \mathrm{p}<0 \cdot 02 ;{ }^{* *} \mathrm{p}<0 \cdot 01 ;{ }^{* * *} \mathrm{p}<0 \cdot(0) 1 ;$ all compared with control group. $\dagger \mathrm{p}<0.05 ; \dagger+\mathrm{p}<0.001$; both compared with the corresponding subgroup. $\ddagger$ For abbreviations see 'Patients and methods". 
Table 2 HLA antigens and disease severity (results expressed as a percentage)

\begin{tabular}{|c|c|c|c|c|}
\hline & Controls & Mild & Moderate & Severe \\
\hline No & 375 & 26 & 20 & 14 \\
\hline Male/female & & $19 / 7$ & $8 / 12$ & $2 / 12$ \\
\hline $\begin{array}{l}\text { Average duration of } \\
\text { disease, years (mean (SD)) }\end{array}$ & & $7.6(8.6)$ & $10.5(9.5)$ & $20.9(12.9)$ \\
\hline A1 & $33 \cdot 6$ & $42 \cdot 3$ & 45 & $71 \cdot 4^{* *}$ \\
\hline B17 & $11 \cdot 2$ & $15 \cdot 3$ & 15 & $50(\div \div)$ \\
\hline No & 163 & 25 & 18 & 14 \\
\hline DR2 & $25 \cdot 1$ & $28(\dagger)$ & 0 & $7 \cdot 1$ \\
\hline DR4 & $28 \cdot 8$ & 20 & $61 \cdot 1^{*}$ & $35 \cdot 7$ \\
\hline DR7 & $22 \cdot 6$ & 40 & $27 \cdot 8$ & $71 \cdot 4(\dagger)^{* * * *}$ \\
\hline
\end{tabular}

${ }^{*} \mathrm{p}<0.02 ;{ }^{* *} \mathrm{p}<0.01 ;{ }^{* * *} \mathrm{p}<0.001 ;$ all compared with control group.

$\dagger p<0.05 ;+t p<0.02$; both compared with other subgroups.

() indicates not significant if corrected for number of variables tested.

Table 3 HLA antigens and presence of erosions (results expressed as a percentage)

\begin{tabular}{llll}
\hline & Control & \multicolumn{2}{l}{ Bone erosions } \\
\cline { 3 - 4 } & & Present & Absent \\
\hline No & 163 & 39 & 18 \\
Male/female & & $12 / 27$ & $14 / 4$ \\
DR3 & $27 \cdot 6$ & $12 \cdot 8$ & $11 \cdot 1$ \\
DR4 & $28 \cdot 8$ & $46 \cdot 1^{*}$ & $17 \cdot 6$ \\
\hline
\end{tabular}

${ }^{*} \mathrm{p}<0 \cdot 05$ compared with control group and other subgroup.

increased HLA-B17 and DR7 and reduced HLADR2 when compared with patients with moderate and mild disease (Table 2). The group with severe disease also had longer duration of the disease, 20.9 (SD 12.9) years, compared with $10.5(9.5)$ years for moderate disease and $7.6(8.6)$ years for mild disease. HLA-DR4 was associated only with moderately severe and not severe disease. There was a strong female predominance in the group with severe disease, 12 of the 14 being female.

The patients with bone erosions had a significantly higher frequency of HLA-DR4 than those without (Table 3 ). The duration of the disease was similar between those positive and those negative for HLA-DR4, 12.4 (SD 11.3) and 11.1 (11.6) years respectively. Bone erosions were more common in women.

\section{Discussion}

The results of this study confirm the known associations between psoriatic arthritis and HLA antigens A1, B17, B27, and DR7. Clinical subgroups need to be considered in order to elucidate other possible associations. HLA-B38 has been associated with peripheral arthritis, ${ }^{8}$ but we were unable to confirm $\stackrel{\supset}{-}$ this. We did find, however, that HLA-DR7 was significantly increased in this group. We did not measure HLA-Cw6, which is known to be in linkage disequilibrium with HLA-B13, B17, and DR7. It $\vec{O}$ has been suggested that HLA-Cw6 is a prime. marker for psoriasis, and other antigens such as HLA-DR7 serve as additional markers for psoriatic arthritis. ${ }^{11}$

The association of HLA-B27 and psoriatic spondylitis is well recorded. ${ }^{128-14}$ In our patients the strongest association with HLA-B27 was with the spondylitis only group, all of whom were male with sacroiliitis. The association became weaker as peripheral joint involvement was included, implying that in the absence of HLA-B27 there are other disease susceptibility antigens in patients with psoriasis, which predispose to development of axial 0 and particularly peripheral joint disease.

There have been conflicting reports regarding the incidence of HLA-B27 in association with peripheral arthritis. Some report an increased incidence ${ }^{12} 12$ 을 and others no association. ${ }^{8-10}$ The reason for this discrepancy is unclear, particularly as the three positive studies cited had radiological evidence of normal sacroiliac joints in patients with peripheral $\sigma$ arthritis who were HLA-B27 positive. Longitudinal N data would be useful in assessing whether these patients develop sacroiliitis at a later date.

The pattern of arthritis seen may also have a major bearing. When we classified our patients according to the five subgroups described by Moll $\stackrel{\oplus}{\rightleftharpoons}$ and Wright ${ }^{17}$ (Table 4) there was an unusually high incidence of polyarticular as opposed to oligoarticu- $\overline{0}$ lar peripheral joint involvement. This may reflect $\mathbb{D}$ the pattern of referral to a specialist rheumatology unit, where patients with mild or limited arthritis are not necessarily seen. 
Table 4 Disease subgroups* (results expressed as a percentage)

$\begin{array}{lr}\text { No of patients } & 60 \\ \text { Predominantly DIP } & 5 \cdot 0 \\ \text { Arthritis mutilans } & 1 \cdot 7 \\ \text { RA-like } & 10 \cdot 0 \\ \text { Asymmetrical: } & \\ \quad \text { Polyarticular } & 60 \cdot 0 \\ \quad \text { Oligoarticular } & 15 \cdot 0 \\ \text { Spondylitis } & 35 \cdot 0\end{array}$

${ }^{*}$ Moll and Wright classification. ${ }^{17}$

$\mathrm{DIP}=$ distal interphalangeal joint: $\mathrm{RA}=$ rhcumatoid arthritis; polyarticular $=>$ four peripheral joints; oligoarticular $=$ one to four peripheral joints.

Six patients had axial involvement but did not have Rome criteria for spondylitis. Three had cervical spine spondylitis and three had sacroiliitis. They all had peripheral joint disease. Only one of the six was HLA-B27 positive, this patient having unilateral sacroiliitis. There may have been some patients with peripheral joint disease only, with subclinical sacroiliitis or cervical spine involvement, who were not $x$ rayed.

According to our results there may be a subgroup of patients with peripheral arthritis and cervical spine involvement who have an increased frequency of HLA-DR1. The cervical spine may be the only axial involvement, especially if these patients are female and HLA-B27 negative.

Gerber et al reported an association between HLA-DR4 and severe disease. ${ }^{10}$ Using the same method to measure disease severity, we found patients with most severe disease had an increase in HLA-B17 and DR7. This method, however, takes no account of length or activity of disease. For instance, our patients with severe disease had arthritis for 20.9 (SD 12.9) years, which was appreciably longer than the other two groups. These antigens may be associated, therefore, with chronicity rather than severity of disease.

Armstrong et al found HLA-DR3 to be associated with the presence of erosions in a study involving 24 patients. ${ }^{1}$ In contrast, we found the group with erosions to have a significantly higher frequency of HLA-DR4 than those without erosions. HLA-DR4 is associated with seropositive rheumatoid arthritis, ${ }^{15}{ }^{19}$ though there is some controversy as to whether the association is with the presence of arthritis or rheumatoid factor. ${ }^{20}$ Seronegative arthritis has less often been associated with HLA-DR4, though it has been suggested as a marker for disease severity in this group. ${ }^{21}$ The results in our patients, all of whom were seronegative for IgM and IgG rheumatoid factor, would support the concept of
HLA-DR4 being a marker for the development of erosions. If one considers the presence of erosions to be a better marker of disease severity than the total number of peripheral joints involved which may reflect disease chronicity, then HLA-DR4 is also a marker for disease severity.

In conclusion, our male patients tended to have spondylitic disease and the female patients peripheral arthritis, which was more likely to be erosive. Among the many genes that confer susceptibility to psoriatic arthritis we confirmed some of the known histocompatibility associations. In addition. HLA-DR7 may be associated with peripheral disease which is more likely to be chronic and severe, and HLA-DR4 with the development of erosions.

The authors gratefully acknowledge the services of the immunohaematology laboratory. Wellington Hospital, and also thank Mrs R Foss for secretarial assistance and Mrs Jean Wright for typing the manuscript.

\section{References}

1 Brewerton D A, Caffrey M. Nicholls A. Walters D, James D C O. HLA-B27 and psoriasis. Lancet 1974; i: 956-72.

2 Karvonen J, Lassus A. Sievers U. Tiilikainen A. HLA antigens in psoriatic arthritis. Ann Clin Res 1974: 6: 304-7.

3 Green L. Meyers O L. Gordon W, Briggs B. Arthritis in psoriasis. Ann Rheum Dis 1981; 40: 366-9.

4 Tiilikainen A. Lassus A, Karvonen J, Vartainen P. Julin M. Psoriasis and HLA-Cw6. Br J Dermatol 1980; 102: 179-84.

5 White H A, Newcomber V D. Mickey M R. Terasaki P I. Disturbance of HLA antigen frequency in psoriasis. $N$ Engl $J$ Med 1972: 287: 740-3

6 Krain L S, Newcomber V D, Terasaki P I. HLA antigens in psoriasis [Abstract]. $N$ Engl J Med 1973; 288: 1245.

7 Roux H. Mercier P. Maestracci D, et al. Psoriatic arthritis and HLA antigens. J Rheumatol 1977; 4: 64-5.

8 Espinoza L R, Vasey F B. Gaylord S W. et al. Histocompatibility typing in the seronegative spondyloarthropathies: a survey. Semin Arthritis Rheum 1982: 11: 375-81.

9 Murray C, Mann D L. Gerber L N, et al. Histocompatibility antigens in psoriasis and psoriatic arthritis. Evidence of the influence of multiple genes in the major histocompatibility complex. J Clin Invest 1980; 66: 670-5.

10 Gerber L H, Murray C L. Perlman S G, et al. Human lymphocyte antigens characterising psoriatic arthritis and its sub-types. J Rheumatol 1982; 9: 703-7.

11 Armstrong R D, Panayi G S, Welsh K I. Histocompatibility antigens in psoriasis, psoriatic arthropathy and ankylosing spondylitis. Ann Rheum Dis 1983; 42: 142-6.

12 Eastmond C J. Woodrow J C. The HLA system and the arthropathies associated with psoriasis. Ann Rheum Dis 1977; 36: $112-20$.

13 McKendry R J, Sengar D P. DesGroseilliers J P. Dunne J V. Frequency of HLA antigens in patients with psoriasis or psoriatic arthritis. Can Med Assoc J 1984; 130: 411.

14 Beaulieu A D. Roy R. Mathon G. et al. Psoriatic arthritis: risk factors for patients with psoriasis-a study based on histocompatibility antigen frequencies. $J$ Rheumatol $1983 ; 10$ : 633-6.

15 Statsny P. Association of a B cell alloantigen DRw4 with rheumatoid arthritis. $N$ Engl J Med 1978; 293: 869-71.

16 Miehle W. Schattenkirchner M. Albert D, Bunge M. HLA- 
DR4 in ankylosing spondylitis with different patterns of joint involvement. Ann Rheum Dis 1985; 44: 39-44.

17 Moll J M H. Wright V. Psoriatic arthritis. Semin Arthritis Rheum 1973; 3: 55-78.

18 Kellgren J H. Diagnostic criteria for population studies. Bull Rheum Dis 1962; 13: 291-2.

19 Panayi G S, Wooly P H. Batchelor J R. Genetic basis of rheumatoid disease: HLA antigens, disease manifestation and toxic reactions to drugs. $\mathrm{Br}$ Med J 1978; ii: 1326.

20 Panayi G S. Genetic studies of rheumatoid arthritis. In: Immunogenetics. London: Butterworth. 1984: ch 6.

21 Alarcon G S, Koopman W J. Acton R T. Barger B O. Seronegative rheumatoid arthritis; a distinct immunog netic disease? Arthritis Rheum 1982; 25: 502. 